\title{
Design of Calculation Method of Airborne VHF Communication Shielding
}

\author{
Hongbo Liu ${ }^{1,2, \text { a) }}$, Jin Meng ${ }^{1}$, Yuanbo $\mathrm{Di}^{2}$, Junkai Ma ${ }^{2}$ \\ ${ }^{1}$ National Key Laboratory for Vessel Integrated Power System Technology, Naval Univ. of Engineering, Wuhan \\ Hubei 430033, China \\ ${ }^{2}$ College of Electronic Engineering, Naval Univ. of Engineering, Wuhan Hubei 430033, China \\ ${ }^{\text {a }}$ wuhanliuliu@126.com
}

\begin{abstract}
As the communication would be interrupted by the airframe when the aircraft platform communicates with VHF over-the-horizon, the paper adopts the modeling method of computing geometry to conduct reducing-dimension modeling through coordinate transformation and rotation. Through simplifying the complicated shielding problem of airborne over-the-horizon communication, the paper concentrates on the calculation problem of computing geometry to judge whether a point in the plane is in the simple polygon, which can be used in project application scenarios of realtime computation.
\end{abstract}

Key words: Aircraft, VHF, Antenna, Over-the-horizon.

\section{INTRODUCTION}

When the aircraft does some maneuver motions, such as making a turn, the submarine would lose some data while receiving the message from the aircraft. As there are enormous reasons that contribute to this phenomenon, it is quite difficult to quantitatively judge whether there is shielding and whether the space and time of shielding can be predicted. Particularly, when the aircraft platform is involved in the communication network simulation and network performance evaluation, it needs to timely calculate the communication shielding between aircraft and submarine, aircraft and base station, aircraft and aircraft.

In Literature 1, it establishes the airborne antenna shielding mathematical models to solve the Antenna shielding angle based on sopherics and to show the specific influence of antenna shielding on flight track by applying uniform geometry theory of diffraction, and the calculation process is quite complex. Moreover, as for the current airborne antenna and its layout design, there are lot of papers related to analysis on airborne antenna EMC technology and emulation of airplane VHF antenna rational layout, but papers on airborne VHF communication shielding calculation method are few. Therefore, a kind of real-time computation method should be designed.

According to the communication rule of electromagnetic wave, the electromagnetic wave above VHF mainly communicates in a straight line and its diffraction ability is weak when it encounters a barrier. When analyzing the shielding factor of airplane itself, to simplify the problem, provided that there is no wave interference around the aircraft, and such factors as Fresnel Zone is not taken into consideration. The paper only analyses the communication interruption caused by airframe shielding, and comes up with a calculation method to solve this problem.

\section{AIRBORNE ANTENNA SHIELDING COMMUNICATION ANALYSIS}

As the airplane platform space is quite limited and the working environment is different from that of ground platform and ship platform, the antenna installation way would affect the airplane performance. Therefore, when 
conducting antenna layout, such factors as airplane structure, intensity, aerodynamic quality and loading requirement need to be taken into consideration, and the installation of antenna should not affect airplane manipulation and flight performance.

VHF radio is mainly used in A-A and A-G communication, radio antenna is usually non-directional antenna and line-of-sight transmission is the main transmission mode. The VHF radio is usually installed in the belly or the back.

Given that the VHF antenna of airborne platform is installed beneath the airplane, the antenna will be partly shielded, for the height and width of other airplane equipment at the bottom are larger than the airplane antenna, which would result in loss of signal and poor G-A communication effect. Besides, there is altitude difference between ground antenna and airplane antenna, which generates antenna shielding angle. The angle is determined by the track, pitching angle of the airplane and antenna altitude difference, and the antenna shielding angle is the major reason of poor G-A communication effect.

\section{Geographic Coordinate Converted to Orthogonal Coordinate}

If the airecraft is at $\mathrm{O}(\lambda 0, \mathrm{o}, \mathrm{Ao})$, in the reference ellipsoid (WGS-84) coordinate, the communication object of the receiving and transmitting antenna is at $\mathrm{T}(\lambda \mathrm{t}, \mathrm{t}, \mathrm{At})$. As it is shown in Figure 1:

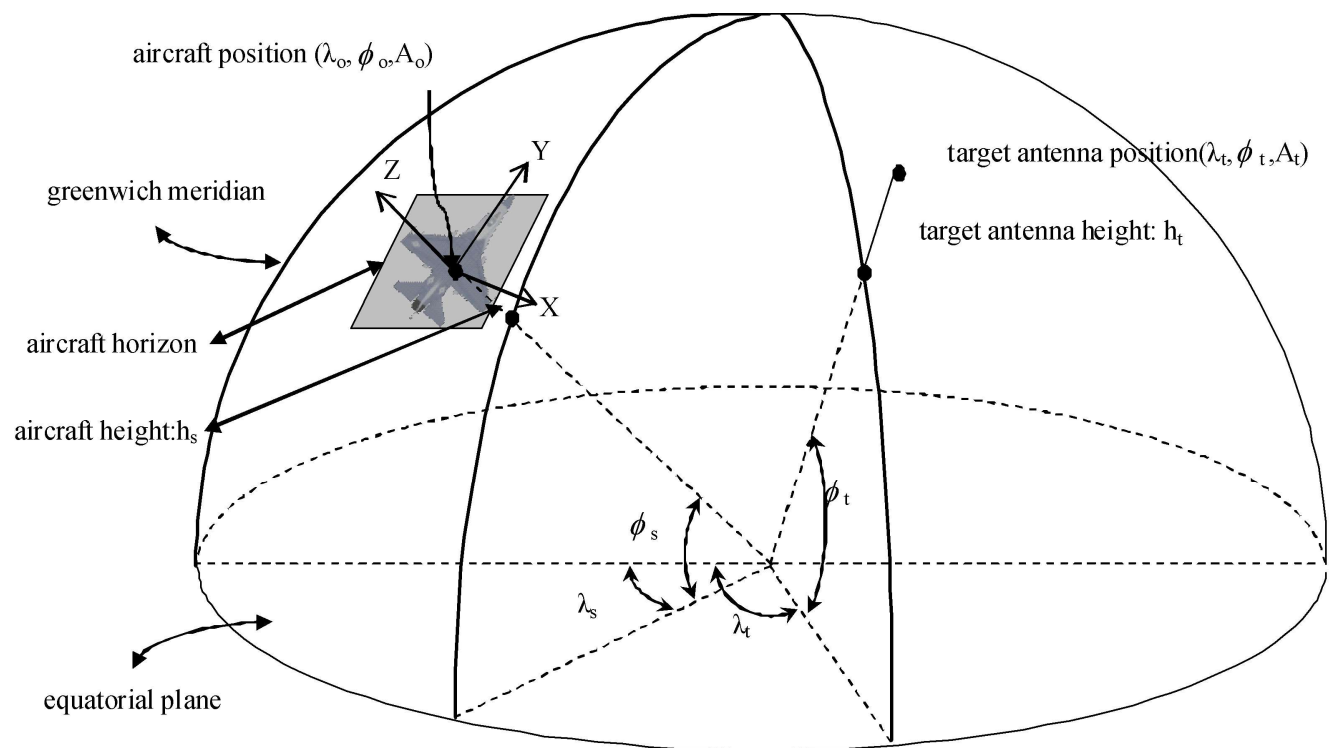

FIGURE 1.Coordinate System of Aircraft and Communication Object

Of these:

$\lambda_{\mathrm{o}}$ is the longitude of the airecraft at $\mathrm{O}$

$\phi_{\mathrm{O}}$ is the height of the aircraft at $\mathrm{O}$

Ho is the height of the airecraft at $\mathrm{O}$

$\lambda t$ is the longitude of the antenna of communication object at $T$

$\phi_{t}$ is the latitude of the antenna of communication object at $\mathrm{T}$

$\mathrm{ht}$ is the height of the antenna of communication object at $\mathrm{T}$.

When the aircraft is at $\mathrm{O}$, cartesian coordinates: $\mathrm{XYZ}$ is established: $\mathrm{X}$ to the east, $\mathrm{Y}$ to the due north, $\mathrm{Z}$ to the sky. When the antenna of communication object is at $T$, the coordinates of this system is (xt,yt,zt), thus,

$$
x_{t}=\left(A_{t}+h_{t}\right) \cos \phi_{t} \sin \left(\lambda_{t}-\lambda_{o}\right)
$$




$$
\begin{gathered}
y_{t}=\left(A_{t}+h_{t}\right)\left[\cos \phi_{o} \sin \phi_{t}-\sin \phi_{o} \cos \left(\lambda_{t}-\lambda_{o}\right)\right]-\Delta K \cos \phi_{o} \\
z_{t}=\left(A_{t}+h_{t}\right)\left[\cos \phi_{o} \cos \phi_{t} \cos \left(\lambda_{t}-\lambda_{o}\right)+\sin \phi_{o} \sin \phi_{t}\right]-A_{o}-h_{o}-\Delta K \sin \phi_{o} \\
\text { Of these } A_{t}=\frac{a}{\sqrt{1-e^{2} \sin ^{2} \phi_{t}}} \\
\Delta K=e^{2}\left(A_{t} \sin \phi_{t}-A_{o} \sin \phi_{o}\right) \\
A_{o}=\frac{a}{\sqrt{1-e^{2} \sin ^{2} \phi_{o}}}
\end{gathered}
$$

Of these, $a$ is the semi-major axis of reference ellipsoid. For WGS-84 earth model, $a=6378.137 \mathrm{~km}$; $e^{2}$ is the square of eccentricity of earth model. For WGS-84 earth model, $e^{2}=0.00669437999013$.

\section{Cartesian coordinate Converted into Aircraft Coordinate}

In the XYZ coordinate system at $\mathrm{O}$, the coordinate of antenna of communication object at $\mathrm{T}$ is (xt,yt,zt). The aircraft coordinate system $X^{\prime} Y^{\prime} Z^{\prime}$ at $O$ is established: $X^{\prime}$ to the right-wing of the aircraft, $Y^{\prime}$ to the aircraft nose, $Z^{\prime}$ to the top of the aircraft. As it is shown in figure 2.

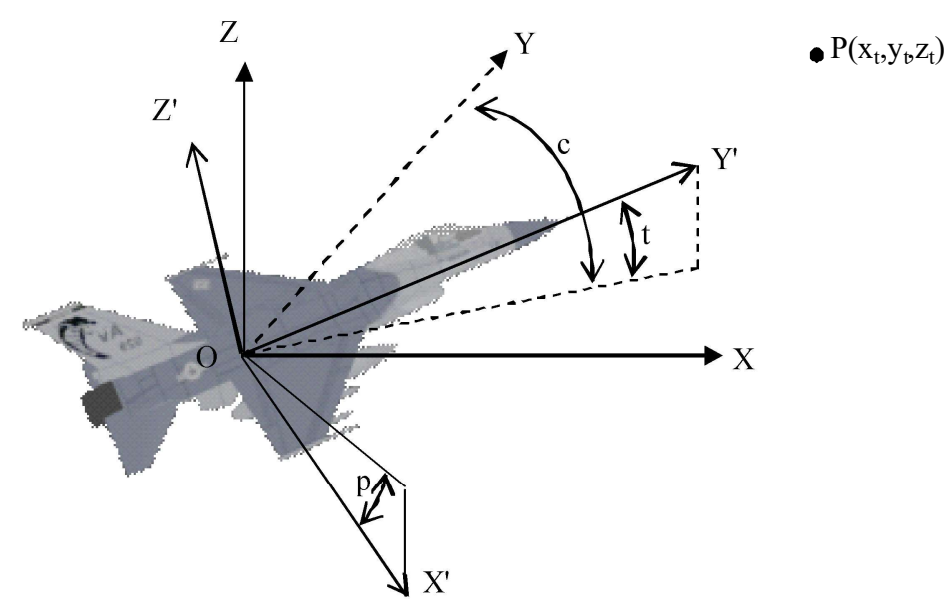

FIGURE 2. Aircraft Coordinate System

Parameters of aircraft attitude (course $\mathrm{c}$, tilt $\mathrm{t}$, roll $\mathrm{p}$ ) are shown below:

(1) $\mathrm{c}$ is the included angle of XOY planar projection of $\mathrm{Y}^{\prime}$ and $\mathrm{Y}$ (from $\mathrm{Z}$ to $\mathrm{O}$, seeing from the clockwise direction, the range is $[0,360)$ )

(2) $t$ is the included angle of XOY planar projection of $Y^{\prime}$ and $Y^{\prime}$ (upward rotation is positive, downward is negative, the range is $[-180,180)$ )

(3) $p$ is the included angle of XOY planar projection of $X^{\prime}$ and $X^{\prime}$ (upward is positive, downward is negative, the range is $[-180,180)$ )

Through the procedures below, the airplane and $\mathrm{T}$ can rotate at the same time, and the relative position of them can be guanranted:

(1), $\mathrm{Y}^{\prime}$ is the axis, when the aircraft and $\mathrm{T}$ rorates $\mathrm{P}$ with the direction contrary to roll angle, and rotating $\mathrm{T}$ to $\mathrm{T} 1$

(2) $X^{\prime}$, as the axis, is at the XOY plane, and then aircraft and T1 rotates t with the direction contrary to pitch angle direction, rotating $\mathrm{T} 1$ to $\mathrm{T} 2$ 
(3) At this time, when $Y^{\prime}$ is at the XOY plane, $Z^{\prime}$ and $Z$ will overlap. Then $Z\left(Z^{\prime}\right)$ as axis, aircraft and T2 rotate $c$ with the direction to $\mathrm{c}$, rotating $\mathrm{T} 2$ to $\mathrm{T} 3$.

Then, $\mathrm{X}$ and $\mathrm{X}^{\prime}$, $\mathrm{Y}$ and $\mathrm{Y}^{\prime}, \mathrm{Z}$ and $\mathrm{Z}^{\prime}$ are overlap, and the aircraft attitude is $(0,0,0)$.

\section{Establishment of Reference Platform}

At the above standard height $\operatorname{Hrp}(\operatorname{Hrp}=20 \mathrm{~m})$, the reference platform is set up. The spot light source is set at the antenna of the belly and the shadow of the aircraft is formed at the reference platform. Seeing the aircraft from the belly to the top, the upward view is shown in the figure 3:

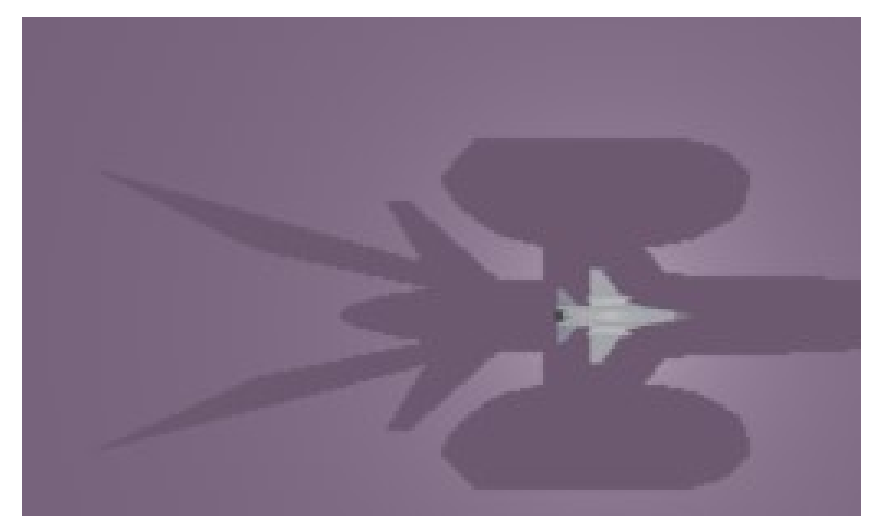

FIGURE 3. Upward View

The calculation method of this paper is applicable to far-field condition. For simplication, the belly antenna is at O. Considering that $\mathrm{O}$ through the radial of $\mathrm{T} 3(\mathrm{x} 3, \mathrm{y} 3, \mathrm{z} 3)$ intersects with the refernece paltform.

In figure 4 , the judgement whether the aircraft is sheltered.

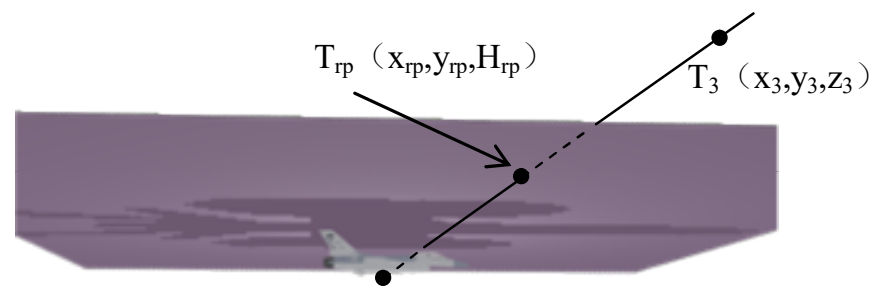

belly antenna

FIGURE 4. Judgement of Aircraft shielding

The radial and reference platform have only one intersection Trp(xrp,yrp,Hrp)

$$
\begin{gathered}
\mathrm{x}_{\mathrm{rp}}=\frac{x_{3}}{\mathrm{z}_{3}} \mathrm{H}_{\mathrm{rp}} \\
\mathrm{y}_{\mathrm{rp}}=\frac{y_{3}}{\mathrm{z}_{3}} \mathrm{H}_{\mathrm{rp}} \\
\mathrm{z}_{\mathrm{rp}}=\mathrm{H}_{\mathrm{rp}}
\end{gathered}
$$

The conditions to judge whether the aircraft is sheltered: 
$\mathrm{z} 3 \leqslant 0$

The radial and reference platform are disjoint, and the aircraft and communication object are not sheltered. Thus, the aircraft itself is not sheltered.

$\mathrm{z} 3>0$

The antenna shielding of aircraft and communication object

\section{Polygon Inside and Outside Decision}

It discusses whether Trp is at the shadow area at the reference platform. If at the shadow area, the shielding happens. For the convenience of calculation, the paper makes the shadow area approximate enclosed polygons. The intersection of adjacent edge and simply polygon has the same apex, and nonadjacent edges are not intersected. As long as it has enough apexes, the accuracy requirement can be met. The problems put forward in this paper can boil down to a basic question: with the given simple polygon $\mathrm{P}$ and Trp, how to judge $\mathrm{P}$ is included in Trp or Trp is in $\mathrm{P}$. The figure 5 presents the judgement whether the aircraft is sheltered.

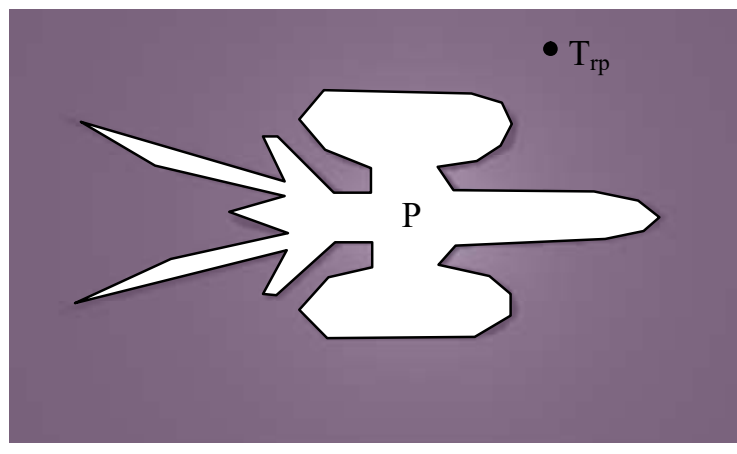

FIGURE 5. Judgement Wheter the Aircraft is Sheltered

\section{CONCLUSION}

When aircraft and other platform conduct VHF or above frequency band communication, the communication interrupt can be casued by aircraft itself. To quantitatively calculate this situation, this paper puts forward a real-time calculation method. This method, by several times of coordinate transformation and rotation to condcut dimensionality reduction on the problem, degenerates the problem into a basic problem of geometry in the plane. The calculation method suggested in this paper is tested in the real system. The antenna shielding between shorebased and aircraft, submarine and aircraft, aircraft and aircraft is quite obvious.

\section{ACKNOWLEDGMENTS}

Fund project: NNSF of China (51422705); Naval Univ. of Engineering Research Development Fund Independent Project; Naval Univ. of Engineering Doctorial Innovation Fund.

\section{REFERENCES}

1. WANG Guo-min, Gu Xiao-peng, Qiu Kai. Effects of Airborne-Antenna Blocking Angle on Air-Ground Communication. Communication Technology,2016,49(12):1724-1727

2. HE Xiao,WU LinZhi. Design of two dimensional nonsingular internal-external cloaks with arbitrary crosssection [J]. Science China.2013,56(7):1373-1383

3. GENG ChangRan, TANG XiaoBin, HOU XiaoXiao, SHU DiYun,CHEN Da.Development of Chinese hybrid radiation adult phantoms their application to external dosimetry[J].Science China.2014,57(4):713-719

4. Jinjin Jiang, Xiaolin Tang, Rui Wu, Haoqiang Lin, Meizhen Qu.Electrochemical performance of polygonized carbon nanofibers as anode materials for lithium-ion batteries [J]. ParticuoloAy, 2013 (11):401-408 
5. TAN Feng-liang, LIU Qing-you, LEI Ting, JIA Shu-jun, DUAN Lin-na. Ferrite evolution during isothermal process in a high deformability pipeline steel [J]. Journal of Iron and Steel Research, International, 2013, 20(7): 89-93

6. ZHANG Shujie,ZHU Axing, LIU Wenliang, LIU Jing, YANG Lin. Mapping detailed soil property using small scale soil type maps and sparse typical samples[J].Chinese Geographical Science,2013 (23)6:680-691

7. Lin-na DUAN,Yu CHEN, Qing-you LIU, Shu-jun JIA, Cheng-chang JIA. Microstructures and Mechanical Properties of X100 Pipeline Steel Strip [J]. Journal of Iron and Steel Research, International, 2014,21(2): $227-$ 232

8. Lanlan Sun, Dongxu Zhao, Meng Ding, Haifeng Zhao, Zhenzhong Zhang, Binghui Li, Dezhen Shen.One-step Synthesis of Shape-controllable Gold Nanoparticles and Their Application in Surface-enhanced Raman Scattering[J]. L. Sun et al: J. Mater. Sci. Technol., 2013, 29(7), 613-618

9. LIU Hu, BAI Zhen-dong, WU Zhe. Method for eatimating geometic characteristics of numerical conceptual aircraft models based on surface elements [J]. Transactions of Nanjing University of Aeronautics\& Astronautics, 2013, 20(2):140-146

10. Yaoyao Li, Donglin Su, Yan Liu,Zhao Yang. The Experimental Study on the Effect of HELP on the Typical Aircraft Antenna. CEEM'2015,HangZhou,2015:116-119 